Int Surg 2012;97:372-373

\title{
International Surgery Author Index to Volume 97
}

Agrelli, Taciana Freitas, 198
Aihara, Arihiro, 6
Akbulut, Sami, 23, 135,
239, 254
Al-Dujaili, Thair M., 150
Aliosmanoğlu, Ibrahim,
245, 305
Amagai, Masayuki, 356
Araki, Kenichiro, 145, 230
Arii, Shigeki, 6
Arii, Kazuo, 351
Arikanoglu, Zulfu, 224,
245, 305
Armato III, Samuel G., 65
Asao, Takayuki, 99, 281
Ashour, Mohamed, 112
Ayten, Refik, 288

Baba, Hiroyuki, 340

Bac, Bilsel, 224

Bando, Toshihiro, 210

Bartlett, Lynne, 112

Bastu, Ercan, 310

Batcholuun, Pandaan, 363

Belliveau, Paul, 285

Bezabeh, Bahiru, 78

Bin, Chen, 104

Bozdağ, Ahmet, 288

Bozdag, Zehra, 135

Braghetto, Italo, 189

Brennan, Nicholas, 140

Brennan, Stephen, 327

Bustamante Toledo, R., 27

Caro, Aleidis, 203

Castaing, Marine, 219

Cavallaro, Mario, 219

Celik, Cem, 310

Cetincakmak, Mehmet Guli, 245

Cetinkaya, Ziya, 288
Chen, Jiye, 71

Chen, Zizi, 71

Chijiiwa, Kazuo, 120

Chkhaidze, Mamuka, 266

Chu, Quyen D., 155

Coles, Maxime J. M., 78

Crema, Eduardo, 198

Cross, Trent, 112

Csendes, Attila, 189

Davanzo, Cristóbal, 189

Davenport, Daniel L., 34

Debandi, Aníbal, 189

Demetrashvili, Zaza, 266

Dietl, Karl-Heinz, 260

Divino, Celia M., 14

Dousei, Tsutomu, 335

Doyon, Laura, 14

Emre, Arif, 135

Endo, Shunji, 335

Engelberger, Stephan, 129

Enkhamagalan,

Tsiiregzen, 363

Ergun, Bulent, 310

Escuder, Jorge, 203

Ferlito, Francesca, 219

Ferraris, Victor, 34

Ferrer Berges, A., 27

Fuchimoto, Yasushi, 356

Fujii, Takaaki, 99, 281

Fujisaki, Hiroto, 356

Fukasawa, Takaharu, 249

Fukuchi, Minoru, 235, 249

Fukumoto, Youji, 276

Fukunaga, Kiyoshi, 315

Furuyama, Takaki, 6

Giedl, Joachim, 260

Girgin, Mustafa, 288
Girgin, Sadullah, 224

Gocho, Takeshi, 360

Gris, Fernando, 203

Guglielminetti, Rachel, 198

Gul, Mesut, 245, 305

Haga, Norihiro, 169, 340

Hassan, Zaki-Udin, 43

Hatanaka, Nobutaka, 335

Hayakawa, Shoichiro, 120

He, Quanyong, 71

Hideshima, Katsumi, 270

Ho, Hung S., 155

Ho, Yik-Hong, 112

Hoffman, Matthew R., 90

Höhler, Florian, 260

Hong, Li, 177

Honjo, Hiroaki, 340

Horiuchi, Katsuhiko, 249

Hoshino, Ken, 356

Hotokezaka, Masayuki, 120

Hou, Ruixing, 321

Hu, WeiLie, 104

Huang, Hai, 104

Huiqing, Xie, 71

Igari, Kimihiro, 6

Iida, Michio, 6

Ikeda, Takuto, 120

Ikeguchi, Masahide, 276

Ikeuchi, Hiroki, 210

Ilhan, Yavuz Selim, 288

Imaizumi, Hideko, 340

Ishibashi, Hiroaki, 270

Ishibashi, Keiichiro,

169,340

Ishida, Hideyuki, 169, 340

Ishida, Yuichi, 360

Ishiguro, Toru, 169, 340

Ishizuka, Mitsuru, 299

Itano, Osamu, 17, 356
Ito, Hiromitsu, 6

Ito, Tetsuya, 340

Javakhishvili, Tamar, 266

Jimenez, Andrea, 203

Ju, Jihui, 321

Kader, Abdul, 276

Kahraman, Aysegul, 239

Kamiike, Wataru, 335

Kanat, Burhan Hakan, 288

Kanat, Zekiye, 288

Kapan, Murat, 224, 245, 305

Kasai, Reo, 6

Kassis, Sebouh Z., 150

Kato, Hatsumi Yano, 270

Kawachi, Shigeyuki, 17, 356

Kawamura, Hideki, 345

Kawano, Tatsuyuki, 340

Kettelhack, Christoph, 129

Khutsishvili, Kakhi, 266

Kindler, Hedy L., 65

Kinoshita, Hiroyuki, 351

Kiriyama, Shinsuke, 249

Kitagawa, Yuko, 17, 356

Kitago, Minoru, 17, 356

Klimkina, Oksana, 43

Ko, Ellen, 1

Kobayashi, Tsutomu, 145,230

Kohsaka, Takayuki, 281

Kong, Xiangbo, 182

Korn, Owen, 189

Koul, Abhinav, 34

Kovzel, Makysym, 327

Kubo, Norio, 145, 230

Kubota, Keiichi, 299

Kuji, Mariko, 345 
Kumagai, Youichi, 6, 340

Kumamoto, Kensuke, 169,340

Kuroda, Tatsuo, 356

Kuwabara, Kohki, 340

Kuwano, Hiroyuki, 99, 145, 230, 235, 249, 281

Li Destri, Giovanni, 219

Li, Hai, 182

Li, Hongyan, 182

Li, Ming, 71

Li, Xiang, 177

Liu, Peng, 293

Liu, Yuefei, 321

Lkhagvabayar, Byadran, 363

Luo, Chengqun, 71

Ma, Hao, 55

Madhoun, Tarek E., 150

Madiba, T. E., 161

Majer, Catalin N., 150

Majeski, James, 95

Masuda, Taiki, 6

Masugi, Yohei, 17

Matsubara, Kentaro, 356

Matsuoka, Hiroki, 210

Misawa, Takeyuki, 360

Miyazaki, Tatsuya, 169

Mnguni, M. N., 161

Mogi, Akira, 281

Mollberg, Nathan M., 65

Moreno Ramiro, J. A., 27

Mori, Kazunari, 351

Muckart, D. J. J., 161

Murphy, Patrick Bates, 285

Mushaya, Chrispen, 112

Nagao, Keisuke, 356

Naitoh, Hiroshi, 235, 249

Nakazato, Kenji, 235

Nariai, Yoshiki, 270

Nasu, Tetsushi, 351

Nguyen, Phuong, 155

Nguyen, Thu, 155

Nishijima, Junichi, 335

Novak, Karel, 260

Obara, Hideaki, 356

Ochiai, Takanori, 6

Oda, Masaya, 17

Oda, Tatsuya, 315
Odaira, Masanori, 356

Odajima, Hajime, 6

Ohara, Kentaro, 17

Ohkohchi, Nobuhiro, 315

Oliva Muñoz, H., 27

Onder, Akin, 224, 245, 305

Ono, Tomojiro, 340

Osaki, Tomohiro, 276

Oshima, Go, 17

Oshiro, Yukio, 315

O-Yurvati, A. H., 1

Ozsurmeli, Mehmet, 310

Paetzel, Christian, 260

Palanci, Yilmaz, 224

Palaniappa, Nandini C., 14

Parsad, Nigel M., 65

Peng, Hao, 71

Pérez Lara, F. J., 27

Petrou, Athanasios, 140

Pipia, Irakli, 266

Preston, Shaun R., 327

Price, Raymond R., 363

Priest, Oliver H., 327

Puleo, Stefano, 219

Qerqadze, Vakhtang, 266

Quintero Quesada, J., 27

Ramaiah, Chandrashe-

khar, 34

Ramos, Marisa de

Carvalho, 198

Rebel, Annette, 43

Ren, Shiyan, 293

Rueda, Juan C., 203

Saito, Hiroaki, 276

Sakamaoto, Michiie, 17

Sakata, Yoshifumi, 351

Sakurai, Shinji, 249

Saleh, Alaa A., 150

Salgia, Ravi, 65

Samdanci, Emine

Turkmen, 254

Sasaki, Ryoko, 315

Sasaki, Shigeru, 145, 230

Schuld, Jochen, 129

Sekine, Joji, 270

Sensakovic, William F., 65

Sergelen, Orgoi, 363

Sheperd, Courtney S., 86

Shiba, Hiroaki, 360
Shibuya, Kazuaki, 345

Shimizu, Atsushi, 351

Shimizu, Takayuki, 299

Shimojima, Naoki, 356

Shimura, Tatsuo, 230

Shinoda, Masahiro, 17, 356

Shoji, Hisanori, 235

Siddiqui, Muhammad

$$
\text { R. S., } 327
$$

Silva, Alex Augusto, 198

Silva, Michael Anthony, 140

Silverstein, Jonathan C., 65

Sobajima, Jun, 340

Soon, Yuen, 327

Soonawalla, Zahir, 140

Spuch, Juan, 203

Stroud, Jason, 95

Suzuki, Hideki, 145, 230

Suzuki, Masaki, 249

Suzuki, Okihide, 340

Tabe, Yuichi, 249

Tacyildiz, Ibrahim, 305

Takahashi, Kazuhiro, 315

Takahashi, Masahiro, 345

Takano, Kiminori, 17

Takaya, Seigo, 276

Takesue, Yoshio, 210

Tanabe, Minoru, 17, 356

Tanaka, Shinji, 6

Tanimoto, Akihiro, 17

Tanioka, Toshiro, 345

Taniyama, Kiyomi, 335

Taskesen, Fatih, 245, 305

Tatebe, Shigeru, 276

Toker, Mehmet, 224

Tomita, Naohiro, 210

Topchishvili, Gega, 266

Trovato, Maria

Antonietta, 219

Tsuji, Yoshitaka, 169

Tsukagoshi, Ritsuko, 249

Tsukamoto, Kanako, 6

Tsutsumi, Soichi, 99, 281

Turkoglu, Ahmet, 288

Uchino, Motoi, 210

Uchiyama, Shuichiro, 120

Ueno, Akihisa, 17

Urade, Masaaki, 296

Valladares, Héctor, 189

Vargas, Gabriela, 363
Vicente, Vicente, 203

Viehl, Carsten Thomas, 129

Vieth, Michael, 270

Vigneswaran, Janani, 65

Vigneswaran, Wickii T., 65

Wada, Wataru, 145, 230

Wakatsuki, Toshiro, 276

Wakiyama, Shigeki, 360

Wamisho, Biruk L., 78

Wang, BangQi, 104

Wang, Kunjie, 177

Wang, Wei, 104, 293

Wang, Yan, 177

Watanabe, Akira, 230

Xia, Yongxiang, 55

Xing, Yuanyuan, 182

Yagi, Masayuki, 6

Yagmur, Yusuf, 23

Yajima, Reina, 99, 281

Yamaguchi, Satoru, 99, 281

Yamaguchi, Shunsuke, 351

Yamaki, Ei, 281

Yamauchi, Amane, 335

Yamauchi, Hayato, 249

Yamazaki, Shigeru, 6

Yanaga, Katsuhiko, 360

Yang, Hongyu, 55

Yang, Luo, 177

Yang, Yuguan, 293

Yilmaz, Mehmet, 135, 239,254

Yilmaz, Sezai, 239, 254

Yin, Zhaoqi, 71

Yoshikawa, Yukinobu, 335

Yoshikawa, Yukinobu, 335

Young, William F., 86

Yuasa, Kazuhisa, 249

Zhang, Gang, 182

Zhang, XiaoMing, 104

Zhang, Xing-Ru, 90

Zhang, Xiwei, 55

Zhang, Zhen-Yong, 90

Zhang, Zhuo, 182

Zhao, Qiang, 321

Zhong, Shi, 71

Zhou, Jianda, 71

Zou, Junjie, 55

Zürcher, Manuel, 129 